\title{
Le(s) discours de l'action collective : contextes, dynamiques et traditions de recherche
}

The Discourse(s) of Collective Action: Contexts, Dynamics and Research

Traditions

Eithan Orkibi

\section{(2) OpenEdition}

\section{Journals}

\section{Electronic version}

URL: http://journals.openedition.org/aad/2002

DOI: 10.4000/aad.2002

ISSN: 1565-8961

\section{Publisher}

Université de Tel-Aviv

\section{Electronic reference}

Eithan Orkibi, «Le(s) discours de l'action collective : contextes, dynamiques et traditions de recherche », Argumentation et Analyse du Discours [Online], 14 | 2015, Online since 09 April 2015, connection on 23 September 2019. URL : http://journals.openedition.org/aad/2002 ; DOI : 10.4000/ aad. 2002

This text was automatically generated on 23 September 2019.

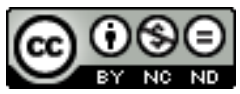

Argumentation \& analyse du discours est mis à disposition selon les termes de la licence Creative Commons Attribution - Pas d'Utilisation Commerciale - Pas de Modification 4.0 International. 


\title{
Le(s) discours de l'action collective : contextes, dynamiques et traditions de recherche
}

\author{
The Discourse(s) of Collective Action: Contexts, Dynamics and Research \\ Traditions
}

Eithan Orkibi

\section{L'harmonie de dissonance}

Clôturant le chapitre intitulé "A Word about Words» dans son célèbre Rules for Radicals, le militant social Saul Alinsky suggère qu'au vu de la centralité du conflit dans la société ouverte, la représentation de la vie démocratique en termes de musique prendrait la forme d'une harmonie de dissonance (1971: 62). Par «harmonie de dissonance " Alinsky désigne une forme de désaccord profond qui va au-delà du dissensus qu'on retrouve dans les grandes controverses, et qu'on pense souvent en termes de discours polémique (Amossy: 2014). Il s'agit plutôt de la contradiction fondamentale entre la réalité sociale d'une part, et la façon dont certains d'entre nous aimeraient la voir, d'autre part. Il s'agit de la contestation constante de l'ordre social et de la structure du pouvoir. Il s'agit du refus de voir dans les conditions sociales une loi transcendantale. L'harmonie de la vie démocratique repose ainsi sur la capacité à faire entendre des voix dissonantes, et ces voix dissonantes s'expriment à travers la parole protestataire et dissidente; à travers les mots de ceux qui agissent, ensemble, pour changer le monde dans lequel ils vivent.

C'est à ce sujet qu'est consacré le présent numéro de la revue Argumentation et Analyse $d u$ Discours, dont l'ambition est double. D'abord, examiner la contribution que peuvent apporter les sciences de langage, en général, et l'analyse du discours (AD) en particulier, à l'étude de ce qui semble (ré)occuper la scène publique ces dernières années dans le monde entier : les mouvements de protestation. Du "printemps arabe » aux manifestations pour des réformes démocratiques à Hong Kong; des tentes dressées 
dans les rues d'Israël au mouvement "Occupy Wall Street» aux États-Unis; du mouvement «Femen » en Ukraine aux manifestations pour et contre le mariage pour tous en France - nous sommes témoins, à l'époque des réseaux sociaux et des nouveaux médias, d'une redécouverte et d'une évolution des formes de mobilisation et des modalités de protestation. Que partagent ces mouvements du point du vue discursif? Quelle est la spécificité du langage qui en résulte?

Plus concrètement, ce numéro souhaite susciter une réflexion sur la parole contestataire au sein de l'AD qui, pour des raisons diverses, n'a pas encore vu se développer, en son sein, le discours protestataire comme domaine de spécialité à part entière. Alors qu'en sciences sociales, et surtout en sociologie et en sciences de la communication, l'aspect "expressif» des mouvements sociaux, c'est-à-dire leur activité discursive et leur production textuelle, est abondamment étudié et analysé, en AD cette activité reste relativement marginale, et souvent conçue comme faisant partie du champ plus large du « discours politique ». Or, comme cette introduction souhaite le montrer, le discours protestataire est caractérisé par certaines spécificités qui justifient qu'on l'aborde comme un terrain distinct d'exploration. Ainsi, la seconde ambition de ce numéro est de relancer une interrogation sur la parole protestataire propre à l'AD, mais qui ne soit pas pour autant isolée de ce qui se pratique dans d'autres disciplines.

Pour ce faire, nous commençons par délimiter notre objet en nous focalisant sur le discours qui accompagne, qui anime et qui émerge de ce qu'il convient d'appeler "l'action collective", à savoir la pratique sociale destinée à contester et modifier certains aspects de la réalité sociale. La première partie de cette introduction se propose d'offrir quelques définitions de travail afin de rendre plus concrets le propos et l'objectif du présent numéro. Nous allons introduire le terme d' " action collective », et discuter ses rapports avec le "discours ». La seconde partie offre un aperçu général de la façon dont le discours de l'action collective est théorisé et analysé dans trois traditions de recherche: la sociologie de l'action collective, la rhétorique des mouvements sociaux et l'école française d'analyse du discours. Nous soulignerons les apports uniques de chaque tradition à l'étude du discours protestataire tout en espérant de la sorte contribuer à la formation d'une approche d'AD à l'étude du discours de l'action collective à la fois autonome et distincte, et ouverte aux dialogues interdisciplinaires.

\section{Définitions}

\section{Le terme d'action collective}

Le terme d'«action collective» s'est implanté dans les sciences sociales à partir des années 1960. Son existence manifeste l'intérêt croissant que la sociologie, la psychologie sociale et les sciences politiques portent aux groupes, de types et de proportions différentes, qui agissent en faveur d'une cause définie. Ce phénomène social - des individus collaborant les uns avec les autres, de manière plus ou moins volontaire et coordonnée, sous la forme d'une collectivité de caractère plus ou moins organisé, dans l'objectif de promouvoir et de défendre leur intérêt (et parfois celui des autres) - semble typique des régimes démocratiques contemporains. Peut-on imaginer la citoyenneté moderne sans cet espace libre de participation sociale et politique dénommé « la société civile $~^{1}$, où les acteurs sociaux - associations, organisations non- 
gouvernementales, syndicats ou groupes d'intérêt - interviennent, agissent, résistent et luttent? Peut-on concevoir la promotion et la réalisation d'un changement social, comme celui du mariage pour tous, sans l'intervention, sur la scène publique, d'un mouvement social qui réunit autour de lui un ensemble de forces sociales idéologiquement marquées et pratiquement engagées en faveur d'une notion novatrice ? Et comment penser à une réforme économique majeure programmée par le gouvernement, comme le Contrat première embauche, sans la réaction contestataire énergique des citoyens concernés, sous la forme d'un mouvement de protestation, de manifestations et défilés, d'arrêts de travail ou de grèves? Il semble que, même si elle apparaît encore à certains comme non-conventionnelle, l'action collective est à l'époque actuelle socialement institutionnalisée comme mode légitime de participation politique. Rien n'est plus logique, dirait-on, que d'agir ensemble afin d'aboutir à un objectif commun.

Or, ce qui paraît évident en principe l'est moins dans la réalité. C'est en 1965 que l'économiste américain Mancur Olson, père de l'approche rationnelle de l'étude des mouvements sociaux, constate que

En réalité, le cas des très petits groupes mis à part, à moins de mesures coercitives ou de quelque autre disposition particulière les incitant à agir dans leur intérêt commun, des individus raisonnables et intéressés ne s'emploieront pas volontairement à défendre les intérêts du groupe (Olson 2011 [1965] : 12).

Il semble que les gens ne s'engagent pas facilement dans un groupe d'action conforme à leurs intérêts et ne partagent pas aisément leur énergie et leurs ressources. Souvent, le choix le plus évident de l'acteur rationnel est d'éviter toute participation à l'action en raison du temps et du coût de l'engagement social - surtout s'il peut bénéficier du résultat de l'action sans y prendre part. Comment expliquer alors la fréquence de l'action collective et le renouvellement constant de ses modalités ? Ce paradoxe, que la sociologie de l'action collective traite en termes de «choix rationnel» et de «free rider" (ticket gratuit) $)^{2}$, nourrit depuis les années 1960 la réflexion autour des « logiques » de l'action collective, à savoir des raisons qui poussent l'individu à y participer.

Il ne faut cependant pas que le terme de "choix rationnel» nous conduise à ne comprendre la participation à l'action collective que comme le résultat de calculs coûtbénéfice ou de considérations sacrifice-intérêt. En fait, lorsqu'on parle des «logiques » de l'action collective, on ne néglige pas le système de valeurs et de croyances, la perception de la réalité sociale et les enjeux psychologiques qui sous-tendent les dynamiques de mobilisation. L'analyse des motivations épistémiques, émotionnelles et culturelles qui amènent à l'action collective permet d'en cerner la dimension dite " subjective ", à savoir, les « récompenses » non-matérielles mais plutôt psychologiques et culturelles dont bénéfice l'individu lorsqu'il s'engage. Ces motifs, qualifiés souvent d'« incitations sélectives ", sont à la base d'une perspective psychologique et culturelle qui, depuis les années 1980, permet de penser la participation de l'individu à l'action collective en termes de solidarité avec ses pairs ou d'effet de mode (bandwagon effect Hirsch 1986), ou encore de tendance à s'unir et serrer les rangs face aux adversaires de son groupe d'appartenance (Klandermans 1984). Prenant en compte de telles dimensions et considérant le désir exprimé par les acteurs de modifier la réalité sociale, les sciences sociales traitent ainsi de l'action collective non seulement comme d'une procédure technique permettant de réaliser certains objectifs, mais comme d'une 
pratique sociale qui comprend des enjeux identitaires, culturels et idéologiques très larges.

Ainsi, notre point de départ consiste à reconnaître ces deux aspects du terme d'action collective. D'un côté, le fait empirique, c'est-à-dire la collectivité mobilisant efforts et ressources afin de modifier certains éléments de la réalité sociale ou de résister à un changement promu par d'autres, usant pour ce faire de différentes modalités d'action qui vont au-delà des processus politiques de routine. Cette action peut être précise et ponctuelle, comme la lutte contre le CPE, mais elle peut également prendre la forme d'un mouvement social plus large, qui se disperse dans l'espace et dans le temps, comme le mouvement pour l'égalité de $\mathrm{LGBT}^{3}$. De l'autre côté, le fait symbolique, c'està-dire le rôle que joue l'action collective dans la façon dont on pense et parle de la réalité sociale. Il s'agit du fait que l'action collective permet, par son existence, de revoir des ordres de priorité, des valeurs, des idées reçues... En bref : elle amène non seulement un changement concret de la réalité sociale, mais aussi une modification dans la perception de cette réalité. Comme le suggèrent Ron Eyerman et Andrew Jamison, les mouvements sociaux peuvent être conçus comme des "espaces publics provisoires, des moments de création collective qui fournissent aux sociétés des idées, des identités, et même de des idéaux » $(1991: 4)$.

\section{Discours et action collective : spécificité, contextes et dynamiques}

«Producteurs de significations ", pour reprendre la formule de Daniel Cefaï (2001:12), les mouvements d'action collective sont amplement dépendants de la communication verbale et symbolique. La raison en est simple : quelle récompense pourrait accorder un mouvement aux militants? Quelle sanction peut être utilisée contre ceux qui font défection et abandonnent la lutte au milieu de l'action? Quelle légitimation a le mouvement d'exister, en dehors de la volonté et de l'engagement des individus? Les outils de travail des mouvements d'action ne sont pas comparables aux instruments dont disposent les institutions officielles. C'est le manque quasi-total d'autorité reconnue, de compétences bureaucratiques et de moyens organisationnels, qui transforme le discours en « agent (agency) principal par le biais duquel les mouvements sociaux accomplissent différentes fonctions qui leur permettent d'exister, de réagir à l'opposition et, peut-être, de réussir à promouvoir (ou à résister à) un changement " (Stewart 1980 : 299).

Mais la centralité du discours va plus loin que la gestion de l'action. La constatation que les mouvements sont "producteurs de signification » implique aussi une vision plus large qui dépasse les préoccupations immédiates telles que le recrutement d'adhérents ou la résistance à l'opposition. Il s'agit de comprendre le discours de l'action collective comme source de modifications à l'intérieur de ce que Michael McGee (1980) dénomme «la structure idéographique » de la société : le rôle joué par les mouvements d'action collective dans l'évolution et la transformation du vocabulaire et du discours qui gèrent le système des normes et des valeurs de notre société. Ainsi, ce qu'on dénomme ici "discours» de l'action collective, va bien au-delà du moment ponctuel ou d'une mobilisation précise, et renvoie, en fait, à plusieurs contextes de création, de diffusion et d'impact. 


\section{Le discours issu de l'action}

Sur le plan diachronique, nous pouvons distinguer entre trois contextes principaux. Le premier, et le plus évident, peut être qualifié de « discours de mobilisation ». Il s'agit de l'ensemble des énoncés, textes écrits et oraux, produits par l'action collective durant l'action, et au service de l'action, des premières étapes de son émergence et jusqu'à sa dispersion finale. Dans cette catégorie, l'on peut trouver les dispositifs et genres «traditionnels» du discours protestataire: appels et déclarations, mots d'ordre et slogans, graffiti et affiches, aussi bien que des comportements symboliques de toutes sortes (comme actions directes, profanation de symboles ou performance artistique). Toute sorte de parole ou de comportement porteur de message et visant à permettre l'action peut être mise dans cette catégorie dans la mesure où elle répond aux soucis persuasifs et organisationnels de l'action, comme diffuser des arguments, répondre aux adversaires ou motiver les militants.

Un deuxième contexte peut être qualifié, d'après Dominique Maingueneau et Frédéric Cossutta (1995) de « discours constituant de l'action collective»: ce sont les produits discursifs «qui donnent sens aux actes de la collectivité » (113). Il s'agit, dans le cas spécifique de l'action collective, des textes qui offrent au mouvement sa raison d'être, et qui sont reconnus comme tels dans le mouvement et autour de lui : textes de fondation, comme les manifestes ou les chartes, ou textes de référence, comme une liste de revendications ou un programme politique. Dans cette catégorie on peut également compter les textes produits sans rapport avec le mouvement, mais qui sont canonisés par lui en raison de leur efficacité symbolique : un ouvrage poétique, un proverbe, ou une chanson. C'est par exemple le cas de Nous triompherons ( We Shall Overcome ») du Mouvement des droits civiques aux États-Unis.

Un troisième contexte peut-être qualifié de "discours rétrospectif ». Il s'agit d'un discours écrit après coup où des leaders et des militants décrivent ou analysent certains aspects de leur mobilisation. Même s'il n'est pas forcement lié au déroulement de l'action, il en est issu, et participe à la construction de son sens. Ce contexte comprend le "travail d'interprétation " " accompli par les acteurs eux-mêmes au moment de l'action et lorsqu'elle est arrivée à terme, c'est-à-dire le processus au cours duquel ils donnent sens à leur mobilisation et élaborent une réflexion introspective par rapport à leur démarche. Et comme le montrent les travaux réunis par Anna Reading et Tamar Katriel (2015), la négociation - sinon la lutte - autour du sens d'une action continue bien après qu'elle ait pris fin, surtout lorsque cette action devient l'objet d'un travail de mémoire et de commémoration qui détermine l'héritage historique, social et culturel des mouvements passés.

\section{Le discours suscité par l'action}

Alors que ces trois contextes - le discours constituant, le discours de mobilisation, et le discours rétrospectif - représentent différentes circonstances d'énonciation et différentes fonctions communicationnelles du discours produit par l'action collective, il convient également d'inclure sous l'étiquette de «discours de l'action collective » le discours suscité par elle. Il s'agit, en premier lieu, du discours provenant des adversaires, qui se trouvent tenus de réagir. Cette catégorie, dont les acteurs constituent - bon gré mal gré - ce que la psychologie sociale de l'action collective qualifie de «système conflictuel» (conflict system) du mouvement (Klandermans 1997 : 
10), comprend deux types d'acteurs. D'un côté, les forces sociales mobilisées contre la cause défendue par le mouvement, qu'on appelle souvent contre-mouvement, et de l'autre côté, les institutions officielles qui représentent l'ordre établi menacé. La réciprocité entre le mouvement et son système conflictuel est très marquée sur le plan discursif, dans la mesure où le premier s'emploie à réfuter les arguments des adversaires (McCaffrey \& Keys 2000), à renouveler constamment ses propres arguments (Darsey 1991) ou à résister aux attaques lancées contre lui (Vanderford 1989). Ainsi, il importe de voir ici non seulement comment le discours de l'action collective suscite le discours des adversaires ou comment il est modelé par lui, mais aussi comment, au cours du temps, l'échange antagoniste entre un mouvement et son système conflictuel est somme toute dialectique et par cela permet de renouveler le discours public autour des questions qui divisent la société (Cathcart 1972).

Une autre catégorie de discours suscité par un mouvement est celui qui provient des représentations sociales qu'on en donne. Il s'agit, en l'occurrence, de la couverture médiatique de l'action collective qui, en temps réel, diffuse ses messages et en modèle la perception publique (Gamson \& Wolfsfeld 1993). Mais il s'agit aussi des représentations ultérieures, qui participent également à l'élaboration des attitudes envers l'action collective: c'est la représentation culturelle de l'action, comme les descriptions photographiques ou filmographiques d'une mobilisation importante (DeLuca \& Peeples 2002), qui influence les modalités par lesquelles (si cela s'effectue) un certain mouvement entre dans la mémoire collective et l'imaginaire social. Enfin, il faut mentionner le discours scientifique autour de l'action collective qui, loin d'être isolé et écarté du champ, est de plus en plus reconnu pour les rôles qu'il joue dans la formation des perceptions publiques du mouvement (Could 2011).

Ainsi, ce qu'on appelle ici « discours de l'action collective » comprend, en premier lieu, la parole et le comportement symbolique d'une collectivité qui agit en faveur d'une cause ou en opposition à un certain changement, comme les slogans ou les discours des militants. Il s'agit, de plus, du discours qui précède l'action, qui la constitue et qui y fait des références intellectuelles ou symboliques, comme le manifeste mais aussi le discours postérieur à l'action, celui où les militants ou la collectivité la décrivent, reflètent, interprètent et commémorent une fois qu'elle est perçue comme achevée. Nous nous sommes également intéressés, dans ce cadre, au discours suscité par l'action, surtout lorsqu'il intervient, dialogue et interagit avec elle: le discours de l'action opposée, comme l'attaque d'un contre-mouvement, ou le discours institutionnel visant à restreindre l'agitation sociale, mais aussi le discours qui en produit des représentations sociales et façonne son image publique, comme la couverture médiatique, la représentation culturelle et l'analyse scientifique. Si chacun de ces espaces discursifs est autonome est conditionné par les enjeux et les contraintes de ses propres contextes et fonctions, il n'en reste pas moins que les rapports entre les différents discours de l'action collective ne sont pas seulement étroits, mais également symbiotiques. 


\section{Traditions}

\section{Sociologie de l'action collective}

La centralité du discours dans et autour de l'action collective n'est pas ignorée des disciplines scientifiques au sein desquelles elle constitue tout naturellement un objet essentiel de recherche, et notamment en sociologie et en psychologie sociale des mouvements sociaux. Dans ces deux domaines, on peut désormais constater depuis les années 1980 un «tournant culturel ». Aux approches structuralistes de l'étude de la mobilisation s'ajoute alors une perspective plutôt constructiviste qui, sous l'influence du tournant linguistique dans les sciences sociales, s'intéresse à la formation des attitudes, des motifs et des significations par l'action collective (Jasper 2010). L'attention se porte vers la dimension expressive de l'action collective et la production textuelle et verbale au sein des mouvements sociaux. C'est essentiellement autour de l'analyse des «cadres" (frames) que ce courant se manifeste et se développe depuis les années 1990 (Cefaï 2011 : 11). Étendant les notions proposées par Erving Goffman dans Frame Analysis (1974) à l'étude des mouvements sociaux, cette approche considère qu'une grande partie de l'activité au sein d'un mouvement social consiste en «la production de signification " et la construction des cadres de l'action collective, à savoir « des ensembles de croyances et de significations » qui donnent sens à l'action, attribuent une signification à la mobilisation, et légitiment l'effort commun (Snow $2001: 28)$.

C'est en élaborant des cadres qu'un mouvement formule une revendication sociale, en dévoilant la nature problématique de certaines conditions sociales ("cadre de diagnostic »), en indiquant une solution (" cadre de pronostic »), tout en soulignant les motifs et les justifications à s'engager («cadre motivationnel»). Et c'est à travers le travail de cadrage qu'un mouvement gère l'opération de mobilisation, en ajustant les cadres (frame alignement) en fonction de différents groupes d'auditoires qu'il convient d'unifier pour la lutte (frame bridging); afin d'en souligner les enjeux idéologiques, éthiques et normatifs des objectifs (frame amplification) ; ou pour élargir la portée d'une revendication précise en montrant son rapport avec d'autres revendications, aspirations et contextes (frame extension) (Benford \& Snow 2000), et ceci dans une situation compétitive qui exige de défendre ses cadres et de miner ceux des adversaires (McCaffrey \& Keys 2000).

Cet intérêt pour le travail de cadrage comme production de sens n'est pas limité aux problèmes et revendications. Le tournant culturel a conduit les chercheurs à examiner aussi le processus de cadrage des identités dans les mouvements d'action collective, considérant que " les constructions identitaires, qu'elles soient intentionnelles ou non, font partie intégrante du travail de cadrage dans tous les mouvements sociaux » (Hunt, Benford \& Snow 1994: 185). Cette construction se fait en fonction de «champs identitaires" (identity fields): le champ protagoniste (les militants et leurs collaborateurs) aussi bien que le champ antagoniste (opposants et adversaires - ibid. : 193-199).

Et enfin, comme en témoigne l'ouvrage récent de l'un des spécialistes le plus reconnus dans le domaine, Sidney Tarrow, The Language of Contention: Revolutions in Words (2013), l'intérêt des sciences sociales pour les dimensions discursives de l'action collective ne se limite pas à l'analyse des cadres d'une action précise. On s'attache également à 
l'évolution complexe des vocabulaires, des modes de raisonnements et des conventions langagières dans la société civile. Ces dernières sont souvent catégorisées comme des "répertoires discursifs » qui se développent au fil des années à travers le rapport dialogique, voire conflictuel, du mouvement avec ses différents interlocuteurs (Steinberg 1999: 750). Dans le même ordre d'idées, en mobilisant la notion de " répertoire d'actions » de Charles Tilly ${ }^{5}$, Juliette Rennes suggère en 2011 de jeter un regard critique sur le "répertoire d'arguments", et d'examiner le "processus de convergence ou de divergence argumentative » dans le discours de l'action collective.

\section{Rhétorique et communication}

Or, il est important de noter ici que l'intérêt exprimé par les sociologues envers l'analyse discursive de l'action collective depuis les années 1980 fait écho à l'immense travail consacré à la parole protestataire dans les sciences de langages et de la communication à partir des années 1960. Si en 1952, le théoricien américain Leland Griffin encourage ses collègues à explorer la "rhétorique des mouvements sociaux " pour renouveler la théorie rhétorique, vers la fin des années 1960, avec les vagues de protestations, c'est une véritable fièvre de recherche sur la persuasion contestataire qui s'empare des États-Unis. Une grande partie de ce courant, dont l'émergence et l'évolution sont décrits dans le numéro 11 d'Argumentation et Analyse du Discours (Orkibi 2013a), est consacrée aux fonctions de la rhétorique dans la gestion de l'action collective, et dans ce sens, ce courant se pose des questions d'ordre sociologique, voire organisationnel: comment rédiger une revendication sociale, recruter des militants, enthousiasmer les manifestants, faire adhérer les masses, et réagir aux opposants (Simons 1971; Bowers \& Ochs 1971), ou encore comment consolider l'identité collective et renforcer la solidarité des militants (Gregg 1971).

Ce courant, plus sensible aux aspects textuels et discursifs de la communication, souligne, dès le départ, les traits caractéristiques de la parole contestataire, dont, notamment, ses objectifs et sa nature polémique. Il s'agit du fait que, souvent, l'action collective émerge là où les arguments " ordinaires » n'ont aucun effet, au moment où celui qui a essayé, en vain, de changer la réalité à travers les mots, doit maintenant passer à l'action; là où ceux dont l'accès à l'opinion publique a été préalablement limité utilisent d'autres modalités pour se faire entendre, comme la provocation, les ultimatums, la profanation de symboles, les mots obscènes... En d'autres termes : tout ce qui va au-delà, et en sens contraire, des processus réguliers de communication dite "constructive». En fait, c'est la nature conflictuelle et coercitive de la parole contestataire qui a poussé les chercheurs non seulement à réexaminer les fonctions de la rhétorique dans le contexte d'agitation sociale, mais aussi à réévaluer les présupposés et les fondements de la théorie rhétorique (Haiman 1967; Hogan \& Tell 2006). Une des constatations les plus importantes posait que, au-delà d'argumenter une revendication sociale et de gérer l'action, le discours de l'action collective est l'instrument principal par le biais duquel les militants peuvent résister aux contraintes sociales - un statut défavorable, la marginalité politique, la discrimination médiatique, etc. (Burgess 1968 et 1970).

Ainsi, dans le domaine dénommé "rhétorique des mouvements sociaux", se sont posées des questions très stimulantes sur les enjeux éthiques et herméneutiques de la parole protestataire: quelles sont les modalités au gré desquelles le mot dissident permet de surmonter des conditions défavorables? Comment les mouvements sociaux 
participent-ils au processus de communication persuasive au sein de la sphère publique, tout en contestant cette structure qui maintient les inégalités sociales? Ces aspects du discours protestataire, relativement moins traités par la sociologie des mouvements sociaux, ont justifié la tentative (inachevée) de créer toute une théorie de la rhétorique protestataire au début des années $1980^{6}$. Car, comme se demande Kevin Deluca (1999a: 29), en dehors de cette spécificité, quelle est la différence entre la parole protestataire et les discours des partis politiques ou des entreprise commerciales? Tous parlent pour persuader, tous persuadent pour aboutir à un objectif concret. Or si le discours des mouvements sociaux a une spécificité, c'est bien le fait qu'il propose, par son existence, une alternative au discours ordinaire, il conteste les principes du discours général, il transgresse, par la dissidence, la logique de la réalité sociale. Ainsi, en plus des arguments, on explore aussi la dimension symbolique de l'action directe comme le sit-in ou occupation des locaux (Bosmajian 1971), le comportement symbolique et la rhétorique du corps (DeLuca 1999b), et les déviations et violations des règles entérinées du discours ordinaire, voire " civilisé », dans la sphère publique (Foust 2010).

\section{L'École française d'Analyse du Discours}

Bien que l'action collective et la protestation soient centrales dans la culture française, on ne relève pas dans les tendances françaises d'AD une tradition distincte axée sur les mouvements sociaux. Peut-être faudrait-il un jour se demander pourquoi l'AD voit dans les discours politique ou littéraire des domaines de spécialisation, mais néglige la spécificité du discours qui surgit et se développe dans la société civile. Cependant, même si une tradition à proprement parler ne s'est pas constituée dans le sens où il n'existe pas de communauté de chercheurs spécialisés sur le discours protestataire, le domaine francophone offre une littérature riche et originale sur le sujet. Sans prétendre fournir dans cette présentation partielle un historique de la contribution de l'AD française à l'étude du langage contestataire, nous voudrions évoquer ici quatre bastions qui constituent des points de repères en sciences du langage et en $A D$, et qui sont étroitement liées au sujet du présent numéro.

Il s'agit, d'abord, des travaux constitués autour de l'approche lexicométrique du discours politique, surtout - au départ - au sein du Centre de recherche de lexicologie politique de l'ENS de Saint-Cloud, dont les premiers travaux portaient sur les tracts de mai 1968, et par des linguistes comme Maurice Tournier, qui a consacré une grande partie de sa recherche à l'analyse lexicale et à l'évolution historique du vocabulaire politique, syndical et révolutionnaire. Largement inspiré de ce milieu, et très engagé dans l'analyse historique du discours, Jacques Guilhaumou, "historien du discours » (2013), a puissamment contribué à l'analyse conjointe des textes et des notions, en étudiant l'émergence, les "biographies", et les fonctions contextualisées, de motsthèmes, mots-d'ordre et énoncés politiques, contestataires, et révolutionnaires (Gilhaumou 2006).

Alors que l'approche historique de Jacques Gilhaumou traite des unités lexicales énoncées par des acteurs précis (porte-paroles, 1998) dans des moments (révolutionnaires) délimités (1989), l'approche historique développée par Marc Angenot, qu'on peut qualifier d'histoire rhétorique des idées, s'intéresse aux produits discursifs nés des rapports dialectiques et continus entre différents mouvements idéologiques dans l'histoire (1995). Cette approche se pose des questions sur les 
modalités discursives, les types d'arguments et les figures rhétoriques utilisés dans les grands mouvements idéologiques (socialisme, anarchisme, fascisme, etc.), à travers l'échange conflictuel entre mouvements opposés qui se querellent (2008). Si cette approche historique ne se focalise pas forcément sur des moments précis de mobilisation, ce sont bien les mouvements sociaux, dans le sens le plus large du terme, c'est-à-dire des courants idéologiques qui promeuvent un changement social ou politique ou y résistent, qui sont placés au centre de l'analyse discursive et rhétorique (2000).

Beaucoup plus proches des moments d'action, ou au moins des moments d'émergence et de constitution des groupes d'action, sont les travaux de Marcel Burger sur l'un des genres les plus distinctifs de l'action collective : les manifestes (2002). Ce qui est perçu parfois comme une étape obligatoire mais somme toute procédurale, cérémoniale, voire technique, dans la vie d'un mouvement idéologique ou social, comme une condition préalable qui permet de passer à l'action après avoir exprimé son objectif (Bowers \& Ochs, 1971: 15), s'avère beaucoup plus complexe et dynamique. Il s'agit d'une véritable déclaration d'existence qui accomplit des fonctions très variées: formuler une revendication, constituer un moment historique, revendiquer une identité sociale et servir de référence intellectuelle et symbolique dans la vie du mouvement (Yanoshevsky 2009).

Et finalement, également centré sur l'étude d'un genre distinctif de l'action collective, est l'essai fondateur d'Olivier Reboul sur la rhétorique du slogan, «formule concise et frappante, facilement repérable, polémique et le plus souvent anonyme, destinée à faire agir les masses » (1975 : 42). Arme de combat, efficace à l'écrit comme l'oral, le slogan, selon Reboul, anime l'émotion, établit une distinction nette et simple entre «bien » et «mal», transforme des notions, des idées et des thèses compliquées en formules simplistes, mais compréhensibles et dotées d'un pouvoir de mobilisation.

À part ces quatre domaines des sciences de langage en français (histoire du vocabulaire révolutionnaire, histoire rhétorique des mouvements idéologiques, étude des caractéristiques et des fonctions des manifestes et des slogans), il est peut-être utile de rappeler ici deux domaines propres à l'école française d'AD qui, même s'ils ne s'intéressent pas exclusivement à la parole protestataire, touchent néanmoins aux caractéristiques très marquées du discours de l'action collective.

Il s'agit, tout d'abord, de la nature "violente » ou «combative " de la rhétorique des mouvements sociaux. Deux approches, l'une focalisée sur la violence verbale et les formes langagières telles que l'insulte, l'injure ou la menace (Rosier 2012; Oger 2012), et l'autre sur la structure et les dynamiques de la communication polémique (Amossy 2014), contribuent ensemble à mieux saisir et comprendre la dimension agressive du discours protestataire. On constate, en un premier temps, que les limites contextuelles entre le discours polémique - nous pensons aux grandes controverses et querelles qui divisent la société - et l'agitation sociale ne sont pas toujours claires et qu'en fait, on ne peut pas établir une distinction nette entre le discours polémique qui surgit d'un débat contradictoire et le discours militant qui naît d'un conflit social (Orkibi 2011). Ceci indique, dans un second temps, que les fonctions et les formes du discours polémique suivent une logique similaire à celle du discours de l'action collective : polariser le monde entre «juste » et "erroné », entre «nous» et «eux», (Garand 2007: 14-16); discréditer des adversaires sur la base de leurs personnalités et de leurs positions ; on y retrouve l'implication émotionnelle des orateurs et la montée en tension de l'échange. 
Surtout, nous constatons le fait que le discours protestataire, comme le discours polémique, ne vise pas à persuader, ni à convaincre, mais à convertir (Plantin 2003 : 407) ou, puisqu'il s'agit bien d'une lutte sociale, à vaincre ${ }^{7}$.

Et enfin, en ce qui concerne la nature " collective » du discours de l'action collective, l'AD s'intéresse depuis les années 1970 à ce qu'il convient d'appeler «le locuteur collectif » (Marcellesi \& Gardin 1974 : 17 ; Gardin 1976 : 16-18), à savoir le « nous » ou le «on» qui est considéré dans une situation de communication comme la source du message. Il s'agit, en l'occurrence, de groupes comme les partis politiques ou les syndicats ouvriers, mais aussi de catégories plus larges comme «le mouvement communiste » ou « les Français » (Gardin 2002 : 352-353). Cette notion est partiellement reprise et développée par Dominique Maingueneau (2004) qui, en introduisant la notion d'«hyperénonciateur", établit une distinction entre le locuteur empirique (les individus qui composent le groupe), l'acteur collectif (l'entité sociale, un parti, un mouvement...), et l'hyperénonciateur (entité «transcendante ", à savoir, une catégorie plus large, comme "la gauche» ou "la nation», 118-119). Cette réflexion permet d'utiliser l'une des notions les plus mobilisées par l'analyse du discours, celle d'ethos - à savoir l'image de soi que le locuteur construit dans son discours - pour étudier la présentation de soi des groupes militants. C'est ainsi que la notion d'ethos collectif a été développée récemment pour analyser la construction des identités publiques dans l'action collective, et pour analyser les rapports entre l'identité collective telle qu'elle est construite de l'intérieur et l'image du groupe telle qu'elle est projetée vers l'extérieur dans la rhétorique d'un mouvement de protestation (Orkibi 2009; Amossy 2010 ; Orkibi 2012).

\section{Les contributions de ce numéro}

Offrant des perspectives variées et complémentaires, les contributions de ce numéro reviennent à certains thématiques décrites dans cette introduction, tout en suscitant de nouveaux questionnements et en abordant des problématiques qui exigent un dialogue entre différentes traditions. Aurélien Allouche s'interroge sur les rôles que joue l'argumentation - et plus précisément le travail argumentatif dans le militantisme environnemental - dans la formation et la vie d'un groupe d'action, en montrant comment l'élaboration commune des arguments en faveur d'une cause sous-tend et anime des processus sociaux au sein du groupe même, et dans ses rapports avec d'autres acteurs. Le rapport entre « argument » et « identité » est exploré de manière approfondie par Ida Hekmat, qui examine la façon dont la formation d'une revendication sociale (claims-making activity) visant à ouvrir le mariage aux homosexuel-le-s en France et en Allemagne, suscite un discours identitaire et exige la constitution et l'affichage d'un « nous » des groupes militants et des catégories sociales concernées. Les enjeux identitaires, et leur implications idéologiques et culturelles plus larges, sont également étudiés par Béatrice Fracchiolla, qui prend elle aussi pour cas de figure le mouvement LGBT, cette fois-ci en France - et en se focalisant sur les formes de violence verbale, dans le discours des forces qui s'opposent au mariage pour tous, dont l'activité discursive reflète une véritable lutte de contrôle du discours normatif dans l'espace public.

Toujours sur les enjeux identitaires, l'article d'Anna Giaufret utilise la notion d'ethos collectif pour étudier la présentation de soi d'un groupe militant à caractère 
anarchique et clandestin. C'est en étudiant le cas de Guerilla Gardening à Montréal que Giaufret suggère le terme de "nébuleuse ethotique " pour étudier les constructions d' ethos dans les cas spécifiques des groupes ou mouvements chez qui l'efficacité d'action repose sur la capacité à maintenir une identité flexible et dynamique. Au carrefour de la sociologie des mouvements sociaux et de l'analyse discursive, la contribution d'Erica Guevara porte sur les récits mobilisateurs des militants aux mouvements en faveur de médias indépendants en Amérique latine, tout en examinant l'importance du flou et de l'équivoque dans la construction d'une expérience militante inclusive et la représentation unifiée et commune d'un mouvement très dispersé dans l'espace et dans le temps. Analysant la grève étudiante au Québec en 2012, Geneviève Bernard Barbeau examine les caractéristiques linguistiques et les fonctions sociales des slogans, qui d'un côté reflètent l'hétérogénéité des forces militantes dans le mouvement du «printemps érable ", et de l'autre côté servent d'instrument de mobilisation et de solidification des participants à la lutte.

Reprenant un corpus historique, François Provenzano étudie une topique centrale de Mai 68, celle du slogan "Libérez l'expression », et trace la constitution et l'élaboration de cette topique à travers différents dispositifs, de l'imprimé aux affiches, tout en explorant les rapports dialectiques entre texte et image, et la migration des idées et des comportements discursifs à travers différents milieux et catégories sociales. Clôturant ce numéro, vient la contribution de Nana Ariel qui revient au projet de rédaction du manifeste global dans l'objectif de réunir les mouvements et les mobilisations du début des années 2010. Ce projet, dont l'ambition était de constituer et d'exprimer la voix d'une collectivité globale imaginée, n'a pas seulement démontré les nouvelles contraintes et fonctions de ce genre classique de la rhétorique protestataire qu'est le manifeste, mais aussi permis de voir les nouveaux enjeux des discours de l'action collective à une période où aucun militant, aucune collectivité et aucune lutte ne peuvent plus échapper à l'impact de la globalisation, des nouveaux médias et des réseaux sociaux.

\section{BIBLIOGRAPHY}

Alinsky, Saul D. 1989 [1971]. Rules for Radicals. A Pragmatic Primer for Realistic Radicals (New York : Vintage Books)

Amossy, Ruth. 2010. La présentation de Soi. Ethos et identité verbale (Paris : PUF)

Amossy, Ruth. 2014. Apologie de la polémique (Paris : PUF)

Angenot, Marc. 1995 [1982]. La parole pamphlétaire (Paris : Payot)

Angenot, Marc. 2000. Les grands récits militants des XIXe et XXe siècles. Religions de l'humanité et sciences de l'histoire (Paris : L'Harmattan)

Angenot, Marc. 2008. Dialogues de sourds. Traité de rhétorique antilogique (Paris : Mille et une nuits) 
Benford, Robert D. \& Scott A. Hunt. 1992. « Dramaturgy and Social Movements : The Social Construction and Communication of Power », Sociological Inquiry $26: 1,36-55$

Benford, Robert D. \& David A. Snow. 2000. « Framing Processes and Social Movements : An Overview and Assessment », Annual Review of Sociology 26, 611-639

Bosmajian, Haig A. (éd.) 1971. Dissent : Symbolic Behavior and Rhetorical Strategies (Boston : Allyn and Bacon)

Bowers, John Waite \& Donovan J. Ochs. 1971. The Rhetoric of Agitation and Control (Reading, MA : Addison-Wesley)

Burger, Marcel. 2002. Les manifestes : paroles de combat. De Marx à Breton (Lausanne \& Paris : Delachaux \& Niestlé)

Burgess, Parke G. (1968). « The Rhetoric of Black Power : A Moral Demand ? ", Quarterly Journal of Speech $54: 2,122-133$

Burgess, Parke G. (1970). « The Rhetoric of Moral Conflict : Two Critical Dimensions », Quarterly Journal of Speech $56: 2,120-130$

Cathcart, Robert S. 1972. «New Approaches to the Study of Movements : Defining Movements Rhetorically », Western Speech $36: 2,82-88$

Cefaï, Daniel. 2001. « Présentation », Daniel Cefaï \& Danny Trom (éds.), Les formes de l'action collective. Mobilisation dans des arènes publiques (Paris : Éditions de l'École de hautes études en Sciences Sociales), 9-23

Cloud, Dana. 2011. « The Only Conceivable Thing To Do : Reflections on Academics and Activism », in Seth Kahn \& JongHwa Lee (Éds), Activism and Rhetoric : Theories and Contexts for Political Engagement (New-York : Routledge), pp. 11-24

Darsey, James. 1991. « From 'Gay is Good' to the Scourge of AIDS : the Evolution of Gay Liberaion Rhetoric, 1977-1990», Communication Studies 42 : 1, 43-66

DeLuca, Kevin Michael. 1999a. Image Politics. The New Rhetoric of Environmental Activism (New-York et London : Guilford Press)

DeLuca, Kevin Micheal. 1999b. « Unruly Arguments : The Body Rhetoric of Earth First!, ACT UP, and Queer Nation ", Argumentation and Advocacy $36: 1,9-21$

DeLuca, Kevin Michael \& Jennifer Peeples. 2002. « From Public Sphere to Pbulic Screen :

Democracy, Activism and the 'Violence' of Seatle », Critical Studies in Media Communication $19: 2$, $125-151$

Eyerman, Ron \& Andrew Jamision. 1991. Social Movements : A Cognitive Approach (University Park : Pennsylvania State University Press)

Gamson, William A. \& Gadi Wolfsfeld. 1993. « Movements and Media as Interacting Systems », Annals of the American Academy of Political and Social Science 528, 114-125

Garand, Dominique. 2007. « La fonction de l'ethos dans la formation du discours conflictuel », Marie-Hélène Larochelle (éd.), Invectives et violences verbales (Québec : Presses de l'Université de Laval), 4-19

Gardin, Bernard. 1976. « Discours patronal et discours syndical », Langages 41, 13-46

Gardin, Bernard. 2002. « Locuteur collectif ». Patrick Charaudeau \& Dominique Maingueneau (éds), Dictionnaire d'analyse du discours (Paris : Seuil), 352-353 
Goffman, Erving. 1974. Frame Analysis (New York : Harper)

Gregg, Richard B. 1971. « The Ego-Function in the Rhetoric of Protest », Philosophy and Rhetoric $4: 2,71-91$

Griffin, Leland M. 1952. « The Rhetoric of Historical Movement », Quarterly Journal of Speech $38: 2$, 184-188

Guilhaumou, Jacques. 1989. La langue politique et la Révolution française. De l'événement à la raison linguistique (Paris : Klincksieck)

Guilhaumou, Jacques. 1998. L'avènement des porte-parole de la République (1789-1792). Essai de synthèse sur les langages de la Révolution française (Lille : Presses Universitaires du Septentrion)

Guilhaumou, Jacques. 2006. Discours et événement. L'histoire langagière des concepts (Besançon :

Presses Universitaires de Franche-Comté)

Guilhaumou, Jacques. 2013. "L'engagement d'un historien du discours : trajet et perspectives », Argumentation et Analyse du Discours 11 [en ligne : http://aad.revues.org/1599]

Foust, Christina R. 2010. Transgression as a Mode of Resistance: Rethinking Social Movement in an Era of Corporate Globalization (Lanham, MD : Lexington Books)

Haiman, Franklyn S. (1967). " 'The Rhetoric of the Streets' : Some Legal and Ethical Considerations », Quarterly Journal of Speech $53: 2,99-114$

Hirsch, Eric L. 1986. "The Creation of Political Solidarity in Social Movement Organization », The Sociological Quarterly $27: 3,373-387$

Hogan, J. Michael \& Dave Tell (2006). «Demagoguery and Democratic Deliberation : The Search for Rules of Discursive Engagement », Rhetoric \& Public Affairs 9 : 3, 479-487

Hunt, Scott A., Robert D. Benford \& David A. Snow. 1994. «Identity Fields : Framing Processes and the Social Construction of Movement Identities », Enrique Laraña, Hank Johnston \& Joseph R. Gusfield (éds), New Social Movements: From Ideology to Identity (Philadelphia : Temple University Press), 185-208

Jasper, James M. 2010. «Cultural approaches in the sociology of social movements », Bert Klandermans \& Conny Roggeband (éds), Handbook of Social Movements Across Disciplines (New York : Springer), 59-109

Keucheyan, Razmig. 2009. «Choix rationnel », Olivier Fillieule, Lilian Mahieu \& Cécile Péchu (éds), Dictionnaire des mouvements sociaux (Paris : Presses de Sciences-Po), 108-116

Klandermans, Bert. 1997. The Social Psychology of Protest (Oxford : Blackwell)

Klandermans, Bert. 1984. « Mobilization and Participation : Social Psychological Expansion of Resource Mobilization Theory », American Sociological Review 49 : 5, 583-600

Maingueneau, Dominique. 2004. « Hyperénonciateur et "participation" ", Langages 156, 111-126

Maingueneau, Dominique \& Frédéric Cossutta. 1995. «L'Analyse des discours constituants », Langages 117, 112-125

Marcellesi, Jean-Baptiste \& Bernard Gardin. 1974. Introduction à la sociolinguistique : la linguistique sociale (Paris : Larousse)

McCaffrey, Dawn \& Jennifer Keys. 2000. «Competititve Framing Processes in the Abortion Debate : Polarization-vilification, Frame Saving, and Frame Debunking », Sociological Quarterly $41: 1,41-61$ 
McGee, Michael Calvin. 1980. «'Social Movements' : Phenomenon of Meaning ? ", Central States Speech Journal $31: 4,233-244$

Oger, Claire. 2012. « La conflictualité en discours : le recours à l'injure dans les arènes publiques ", Argumentation et Analyse du Discours 8 [en ligne : http://aad.revues.org/1297]

Olson, Mancur. 2011 (1965). La logique de l'action collective, trad. Mario Levi (Bruxelles : Éditions de l'Université de Bruxelles)

Orkibi, Eithan. 2008. «Ethos collectif et Rhétorique de polarisation : le discours des étudiants en France pendant la guerre d'Algérie ", Argumentation et Analyse du Discours 1 [en ligne : http:// aad.revues.org/438]

Orkibi, Eithan. 2011. « Entre polémique et agitation : la violence verbale dans l'opposition au plan de désengagement de la bande de Gaza », Semen 31, 145-162

Orkibi, Eithan. 2012. Les étudiants de France et la guerre d'Algérie. Identité et expression collective de l'UNEF (1954-1962) (Paris : Syllepse)

Orkibi, Eithan. 2013a. "Critique et engagement dans la rhétorique des mouvements sociaux », Argumentation et analyse du discours 11 [en ligne : http://aad.revues.org/1559]

Orkibi, Eithan. 2013b. « Violence verbale et mouvements sociaux : une approche rhétorique », Béatrice Fracchiolla, Claudine Moïse, Christina Schultz-Romain \& Nathalie Auger (éds), Violences verbales : analyses, enjeux et perspectives (Rennes : Presses Universitaires de Rennes), 55-68

Pirotte, Gautier. 2007. La Notion de société civile (Paris : La Découverte)

Plantin, Christian. 2003. « Des polémistes aux polémiqueurs », Gilles Declercq, Michel Murat \& Jacqueline Dangel (éds), La parole polémique (Paris : Champion), 377-408

Reading, Anna \& Tamar Katriel. 2015. Cultural Memories of Nonviolent Struggles (Baginstoke : Palgrave)

Reboul, Olivier. 1975. Le slogan (Bruxelles : Complexe)

Rennes, Juliette. 2011. « Les formes de la contestation. Sociologie des mobilisations et théories de l'argumentation », A contrario 16, 151-173

Rosier, Laurence. 2012. «Introduction » (Insulte, violence verbale, argumentation), Argumentation et Analyse du Discours 8 [en ligne : http://aad.revues.org/1321]

Simons, Herbert W. 1971. « Requirements, Problems, and Strategies : A theory of persuasion for social movements ", Quarterly Journal of Speech $56: 1,1-11$

Snow, David. 2001. « Analyse de cadres et mouvements sociaux », Daniel Cefaï \& Danny Trom (éds.), Les formes de l'action collective. Mobilisation dans des arènes publiques (Paris : Éditions de l'École de hautes études en Sciences Sociales), 27-49

Steinberg, Marc W. 1999. " The Talk and Back Talk of Collective Action : A Dialogic Analysis of Repertoires of Discourse among Nineteenth-Century English Cotton Spinners ", American Journal of Sociology $105: 3,736-780$

Stewart, Charles J. 1980. «A Functinal Approach to the Rhetoric of Social Movements ", Central States Speech Journal $31:$ 4, 298-305

Tarrow, Sidney G. 2013. The Language of Contention. Revolutions in Words 1688-2012 (New York: Cambridge University Press) 
Taylor, Verta \& Nella Van Dyke. (2004). « ‘Get up, Stand up !’ : Tactical Repertoires of Social Movements ", David A. Snow, Sarah A. Soule \& Hanspeter Kriesi (éds), The Blackwell Companion to Social Movements (Oxford : Blackwell), 262-293

Tilly, Charles. 1995. « Contentious Repertoires in Great Britain, 1758-1834 », Mark Traugott (éd.), Repertoires and Cycles of Collective Action (Durham, NC : Duke University Press), 15-42

Vanderford, Marsha L. 1989. «Vilification and Social Movement : A Case Study of Pro-Life and Pro-Choice Rhetoric », Quarterly Journal of Speech $75: 2,166-182$

Yanoshevsky, Galia. 2009. «Three Decades of Writing on Manifesto : The Making of a Genre », Poetics Today $30: 2,257-286$

\section{NOTES}

1. Sur les concepts de "société civile », voir Gautier Pirotte 2007.

2. J'emprunte cette traduction du terme à la présentation de la théorie du choix rationnel de Razmig Keucheyan (2009).

3. Lesbiennes, gays, bisexuels et transgenres.

4. Nous utilisons ici les termes de Robert D. Benford et Scott A. Hunt (1992: 47-48), dans leur présentation de l'approche dramaturgique à l'action collective.

5. Le terme de "répertoire d'action collective» ou "répertoire de contestation " désigne le choix de modalités d'action et formes de contestation qui est à la disposions d'un mouvement dans un contexte socio-historique donné. Voir Tilly 1995, ainsi que Taylor \& Van Dyke (2004).

6. Voir le numéro spécial de 1980 ( $n^{\circ} 31$, vol. 4) du Central States Speech Journal.

7. Pour une discussion sur la polémicité et la violence verbale dans la rhétorique des mouvements sociaux, voir Orkibi 2013 b.

\section{AUTHOR}

\section{EITHAN ORKIBI}

Université d'Ariel, ADARR 